\title{
The Approximation of the Chemical Reaction Rate by Solving the Integral Equation
}

\author{
D.L. Tsyganov \\ Instituto de Plasmas de Fusao Nuclear, Laboratorio Associado \\ Instituto Superior Tecnic, Av. Rovisco Pais, 1049-001, Lisboa, Portugal \\ Corresponding Author: tdl-tdl@rambler.ru
}

Copyright (C2013 Horizon Research Publishing All rights reserved.

\begin{abstract}
The paper discusses possible methods of approximation of the chemical reaction rate constant for the range of values that lie outside of the experimental temperature range: direct approximation of chemical reaction rate constants obtained by processing experimental values; approximation based on an analytical model of dependence of the integrated process cross-section on energy; and approximation based on the direct solution of the chemical reaction rate constant equation with arbitrary dependence of the integrated process cross-section on energy. The second-order reactions $\mathrm{CH}_{4}+\mathrm{M} \rightarrow \mathrm{CH}_{3}+\mathrm{H}+\mathrm{M}$, $\mathrm{CH}_{3}+\mathrm{M} \rightarrow \mathrm{CH}_{2}+\mathrm{H}+\mathrm{M}, \mathrm{CH}_{3}+\mathrm{M} \rightarrow \mathrm{CH}+\mathrm{H}_{2}+\mathrm{M}$ were explored. To solve the integrated equation, the variational Tikhonov's regularization method was used. It was shown that this method allowed both estimating the threshold energy value and re-establishing the crosssection form. By using the calculated cross-section we can obtain estimated chemical reaction rate constants over a wide temperature range. The data obtained can be used in various calculations in applied fields, in particular, in hypersonic gas dynamics problems, as well as for filling information system databases.
\end{abstract}

Keywords Tikhonov's regularization, Chemical reaction rate constant, Cross-section, Integral equation

\section{Introduction}

Reliable values of chemical reaction rate constants are very often required for calculation of chemical reactors, jet streams of combustion products, designing landing aircraft, etc. In many cases, the temperature range necessary for calculation lies beyond the limits where such chemical reaction rate constants were obtained. For example, a major problem in the design of landing aircraft is providing sufficient thermal protection at a reasonable aircraft weight $[1,2]$. In front of a moving body, an intense shock wave emerges moving with the same speed as the landing aircraft. The shock wave action results in heating the gas and increasing its pressure and density. The temperature immediately behind the shock wave front is tens of thousands degrees, and the pres- sure ranges from $10^{-4} \ldots 10^{-3} \mathrm{~atm}$. at atmosphere entry to hundreds of atm. in the lower layers [1].

The present time, one of the methods used for the interpolation of the chemical reaction rate constants for the temperature range beyond the experimental range based on curve fitting an analytic continuation of the function obtained by processing experimental values.

Another way of the interpolation of the chemical reaction rate constants for the temperature range beyond the experimental range is theoretical calculation of chemical reaction rates. However, despite certain success in theoretical calculations of chemical reaction rates, in general, calculations based on the theory often give rate constants that are significantly different from experimental values. They may be either higher or lower than experimental values by several orders of magnitude. Calculations of kinetic parameters based on molecular dynamic data seem to be quite inefficient. In research practice, such calculations are only justified if molecular dynamics results provide a qualitative explanation of the observed kinetic patterns in the whole reaction class.

Therefore, the method combining the above two methods is gaining ground. This method uses theoretical dependence of real molecular structure and interaction models to describe the functional dependence, and model parameters are further determined based on experimental values of the chemical reaction rate.

The present time, the development of computational methods [3,4] for solving ill-posed problems makes it possible to solve equations for integral cross-section $\sigma(E)$, and then calculate the chemical reaction rate constants over temperature range of interest. Step-by-step, solution of problems by computational methods for solving ill-posed problems is gaining in popularity. Thus, it has been already widely used to solve problems of astrophysics, signal processing, image processing and analysis, CT scanning etc. [3]. Unfortunately, little has been published in the field of chemical kinetics.

\section{Analysis procedures}

\subsection{Computational characteristics}

Calculations were performed using the high-level programming language MatLab [5]. F or the calculations we 
used a computer with dual-core Xeon $(2 \mathrm{GHz}, 10 \mathrm{~GB})$ processor. Calculation took 5 to 6 hours. Functionals were minimized by the conjugate gradient method implemented by MatLab $[6,7]$. Integrals were approximated using the Simpson's formula [8].

\subsection{Initial data and Data analysis.}

The present time, a large array of experimental data has been collected for the rate constants of various chemical reactions. The best-known of these are NIST [9], Kintech Lab [10], Reaction Design [11], Kintecus [12], etc. Approximation of the chemical reaction rate constant in most cases is based on the generalized dependence on the temperature $T: A T^{n} \exp \left(-\frac{E a}{T}\right)$, obtained by Henricus van 't Hoff in 1883 and then Arrhenius in 1989 [13] based on an ingenious hypothesis of the existence of a dedicated subclass of active molecules in the reacting system.

As a rule, the value of the pre-exponential factor $A$, exponent $n$, and activation energy $E a$ are found based on the best match with experimental data over the investigated temperature range by the least-square method [14]. For example, the second-order reaction $\mathrm{CH}_{4}+\mathrm{M} \rightarrow \mathrm{CH}_{3}+\mathrm{H}+\mathrm{M}$ shows considerable scatter of the pre-exponential factor, exponent and activation energy values (Table 1). Despite this scatter of the pre-exponential factor, exponent and activation energy values, the scatter of the absolute chemical reaction rate constant values within the experimental temperature range accounts approximately for $30 \%$ (Fig. 1, a). However, if we extend the dependence of the chemical reaction rate constant proposed by some authors to the temperature range beyond the experimental range of values, we see large (1,000 times or more) scatter of values (Fig. 1, b).

The scatter of the chemical reaction rate constant values in temperature range outside of the experimental (Fig. 1, b) can be explained by the fact that an error in determining the pre-exponential factor $\delta A$, exponent $\delta n$ and activation energy $\delta E a$ values enters nonlinearly. This results in the fact that a relative error $\left|\frac{\delta k(T)}{k(T)}\right|$ of the absolute chemical reaction rate constant value is heavily and nonlinearly temperature-dependent.

$$
\begin{aligned}
& \left|\frac{A T^{n} \exp \left(-\frac{E a}{T}\right)-(A+\delta A) T^{n+\delta n} \exp \left(-\frac{E a+\delta E a}{T}\right)}{A T^{n} \exp \left(-\frac{E a}{T}\right)}\right| \approx \\
& \approx\left|1-T^{\delta n} \exp \left(-\frac{\delta E a}{T}\right)\right|
\end{aligned}
$$

Further, the paper [15] addressed the issue of the apparent activation energy at high temperatures and showed that the slope of the Arrhenius line changes. In the assumption of the Maxwell-Boltzmann distribution, the function of the integrated collision cross-section $\sigma(E)$ close to the reaction threshold is essential for the theoretical calculation of the chemical reaction rate. Behavior of the integrated collision cross-section $\sigma(E)$ function near the threshold can be represented as a power function $(E-E 0)^{m}$. For ionization in collision of heavy particles, $m$ lies in the range of 2 to $3[16,17]$ and electron impact in the range of 1 to 2 [18].
Having calculated the rate constant for the assumption described above, we obtained the following for the two limiting cases:

$$
k(T)= \begin{cases}\left.\frac{8 k_{b} T}{\pi \mu}\right)^{3 / 2} \sigma_{0 \exp }\left(-\frac{E 0}{k_{b} T}\right) \frac{m}{\left(E 0 / k_{b} T\right)^{m-1}}, \frac{E 0}{k_{b} T} \gg 1 \\ \left.\frac{8 b_{b} T}{\pi \mu}\right)^{3 / 2} \sigma_{0} \exp \left(-\frac{E 0}{k_{b} T}\right), & \frac{E 0}{k_{b} T} \ll 1\end{cases}
$$

where $k_{b}$ - Boltzmann constant, $J / K$;

$\mu$ - reduced mass of system, $k g$;

$\sigma_{0}$ - permanent part of the cross-section, $m^{2}$;

$E 0$ - threshold energy, $J$.

The foregoing shows that a direct approximation of the dependence of the chemical reaction rates for the outside-of-experimental temperature region can result in fundamental errors, both in the rate constant value and its dependence on the system parameters as well as in understanding of the reaction mechanism.

\subsection{Solutions. Analysis of results.}

We propose to consider two methods to obtain reliable chemical reaction rate constant values. The first method involves defining model parameters based on experimental chemical reaction rate values for various, more realistic structure and molecule interaction models. The most comprehensive review of models and the formula for the chemical reaction rate constant, as well as recommendations for their use, is presented in [19]. Based on this review, three models were selected and explored for the reactions with the energy threshold.

Model I - The Hard Sphere Model with all relative particle motion energy as input to activation.

$$
\begin{aligned}
& \sigma(E)= \begin{cases}0, & \text { if } E<E 0 \\
\pi R^{2}, & \text { if } E \geq E 0\end{cases} \\
& k(T)=\left(\frac{8 \pi k_{b} T}{\mu}\right)^{1 / 2} R^{2}\left(1+\frac{E 0}{k_{b} T}\right) \exp \left(-\frac{E 0}{k_{b} T}\right)
\end{aligned}
$$

where R - gas-dynamic radius of elastic collision.

Model II - The Hard Sphere Model with the radial component of relative particle motion energy as input to the activation (along the line connecting centers of mass of these particles)

$$
\begin{aligned}
\sigma(E) & = \begin{cases}0, & E<E 0 \\
\pi R^{2}, & E \geq E 0\end{cases} \\
k(T) & =\left(\frac{8 \pi k_{b} T}{\mu}\right)^{1 / 2} R^{2} \exp \left(-\frac{E 0}{k_{b} T}\right)
\end{aligned}
$$

Model III - The Above-Threshold Cross-Section maximum based Model with on the initial cross-section increase and following decrease to zero at high energies.

$$
\begin{aligned}
& \sigma(E)= \begin{cases}0, & E<E 0 \\
B \frac{(E-E 0)^{r}}{E} \exp (-l(E-E 0)), & E \geq E 0\end{cases} \\
& k(T)=B\left(\frac{8 \pi k_{b} T}{\mu}\right)^{1 / 2} \frac{\left(l k_{b} T\right)^{r-1}}{\left(1+l k_{b} T\right)^{r+1}} \Gamma(r+1) \exp \left(\frac{-E 0}{k_{b} T}\right)
\end{aligned}
$$

where $B, r, l$ - model parameters;

$\Gamma$ - Gamma function. 


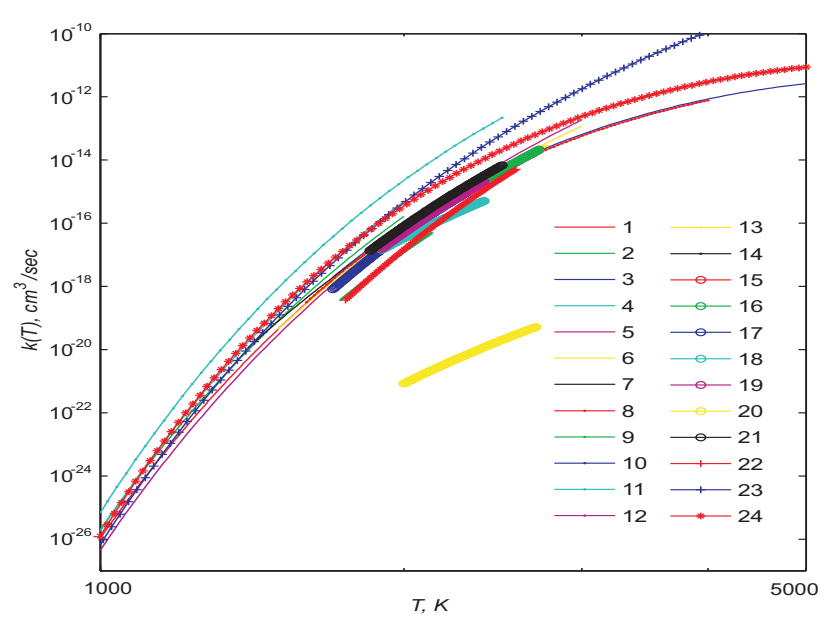

a)

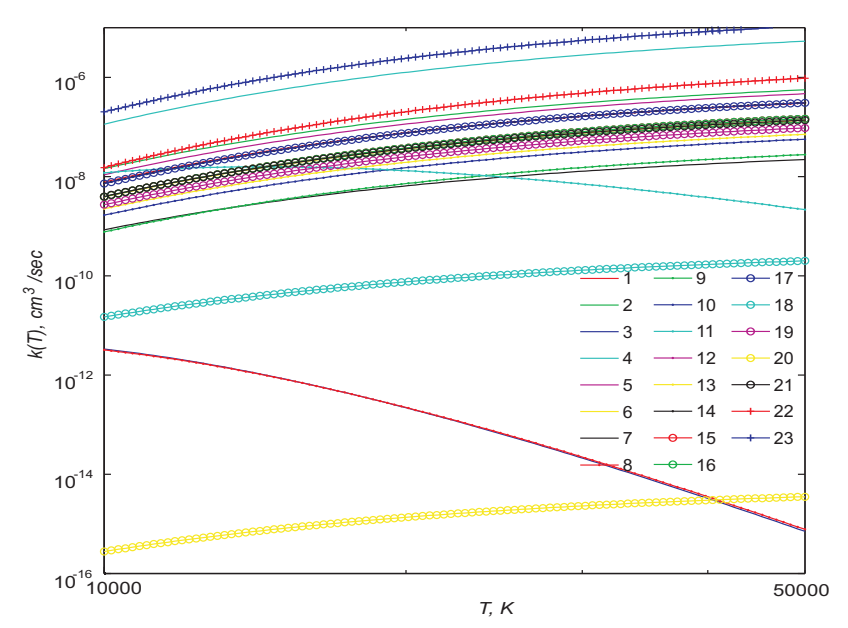

b)

Figure 1. Comparison of the chemical reaction rate constant obtained by various authors (Table 1 ). Reaction $C H_{4}+M \rightarrow$ $\mathrm{CH}_{3}+H+M$ : a - Value of the chemical reaction rate constant inside-of-experimental temperature rang, $\mathrm{b}$ - Approximation of the chemical reaction rate constant outside-of-experimental temperature range. 1 - 1994BAU/COB847-1033-1, 2 - 1994BAU/COB847-1033-2, 3 - 1994BAU/COB847-1033-3, 4 - 1992BAU/COB411-429, 5 - 1992BAU/COB411-429, 6 - 1984WAR197C, 7 - 2000KOI/KUD1-6, 8 - 1993KIE/KUM414-420, 9 - 1992KLE/SUT1786-1793, 10 - 1992DAV/DIR589-596, 11 - 1989STE/SMI923-945, 12 - 1980ROT93, 13 - 1979TAB/BAU63, 14 - 1979ROT/JUS1339, 15 - 1978PEN/SUL279, 16 - 1977HEF/PAR997, 17 - 1975ROT/JUS68, 18 1975GAR/OWE857, 19 - 1975BOW869, 20 - 1973VOM788, 21 - 1971HAR TRO147, 22 - 1971DEA/KIS1718, 23 - 1965PLA/RAB40714080, 24 - mean values.

Table 1. Overview of the pre-exponential factor $A$, the exponent $n$, activation energy $E a$ - for the chemical reaction rate constant of second-order $\mathrm{CH}_{4}+\mathrm{M} \rightarrow \mathrm{CH}_{3}+\mathrm{H}+\mathrm{M}$ it is obtained by various authors.

\begin{tabular}{|c|c|c|c|c|c|}
\hline$A, c m, s e c$ & $n$ & $E a, K$ & Temperature Range, $K$ & According of NIST (Squib) $[9]$ & Reference \\
\hline $7.51 \cdot 10^{-07}$ & 0 & 45,728 & $1000-1700$ & 1994 BAU/COB847-1033-1 & {$[26]$} \\
$1.40 \cdot 10^{-06}$ & 0 & 45,728 & $1000-2000$ & 1994 BAU/COB847-1033-2 & {$[26]$} \\
$8.25 \cdot 10^{23}$ & -8.20 & 59,206 & $1700-5000$ & 1994 BAU/COB847-1033-3 & {$[26]$} \\
$1.40 \cdot 10^{-05}$ & 0 & 48,135 & $1000-2000$ & $1992 B$ BU/COB411-429 & {$[27]$} \\
$1.20 \cdot 10^{-06}$ & 0 & 47052 & $1000-3000$ & 1992 BAU/COB411-429 & {$[27]$} \\
$3.32 \cdot 10^{-07}$ & 0 & 44,525 & $1500-3000$ & 1984 WAR197C & {$[28]$} \\
$4.98 \cdot 10^{-08}$ & 0 & 40,794 & $1400-2500$ & 2000 KOI/KUD1-6 & {$[29]$} \\
$3.41 \cdot 10^{23}$ & -8.11 & 59,085 & $1600-4000$ & 1993 KIE/KUM414-420 & {$[30]$} \\
$6.79 \cdot 10^{-08}$ & 0 & 44,886 & $1730-2130$ & 1992 KLE/SUT1786-1793 & {$[31]$} \\
$1.36 \cdot 10^{-07}$ & 0 & 44,043 & $1790-2320$ & 1992 DAV/DIR589-596 & {$[32]$} \\
$2.17 \cdot 10^{09}$ & -3.73 & 53,670 & $300-2500$ & 1989 STE/SMI923-945 & {$[33]$} \\
$7.85 \cdot 10^{-07}$ & 0 & 46,811 & $1800-2300$ & $1980 R O T 93$ & {$[34]$} \\
$1.68 \cdot 10^{-07}$ & 0 & 43,200 & $1950-2770$ & 1979 TAB/BAU63 & {$[35]$} \\
$7.85 \cdot 10^{-07}$ & 0 & 46,811 & $1800-2300$ & $1979 R O T / J U S 1339$ & {$[36]$} \\
$3.65 \cdot 10^{-07}$ & 0 & 45,367 & $2020-2720$ & 1978 PEN/SUL279 & {$[37]$} \\
$3.65 \cdot 10^{-07}$ & 0 & 45,367 & $2020-2720$ & 1977 HEF/PAR997 & {$[38]$} \\
$7.85 \cdot 10^{-07}$ & 0 & 46,931 & $1700-2300$ & $1975 R O T / J U S 68$ & {$[39]$} \\
$3.82 \cdot 10^{-10}$ & 0 & 32,490 & $2000-2700$ & 1975 GAR/OWE857 & {$[40]$} \\
$2.32 \cdot 10^{-07}$ & 0 & 44,525 & $1900-2400$ & 1975 BOW869 & {$[41]$} \\
$6.61 \cdot 10^{-15}$ & 0 & 31,769 & $2000-2700$ & 1973 VOM788 & {$[42]$} \\
$3.32 \cdot 10^{-07}$ & 0 & 44,284 & $1850-2500$ & 1971 HAR TRO147 & {$[43]$} \\
$2.71 \cdot 10^{-06}$ & 0 & 49,699 & $1750-2580$ & 1971 DEA/KIS1718 & {$[44]$} \\
$2.92 \cdot 10^{-05}$ & 0 & 49,820 & $600-4000$ & 1965 PLA/RAB4071-4080 & {$[45]$} \\
\hline
\end{tabular}

Advantage of first method to be, simplicity of realization. As deficiency it is possible to notice that it is impossible to predict in advance what of (I, II or III) models optimum to used.

The second way of interpolating the chemical reaction rate constant involves calculation based on the integrated cross-section $\sigma(E)$ and the distribution function $f(E)$. If in the simple case

$$
k(T)=\int_{E 0}^{\infty} E^{1 / 2} \sigma(E) f(E) d E
$$

then we must find a way to determine the integrated cross-section $\sigma(E)$, distribution function $f(E)$ and threshold energy $E 0$.

The main problem in using equation (9) to determine the chemical reaction rate constant is a need to define the integrated cross-section $\sigma(E)$ and distribution $f(E)$ functions over a wide energy range, as well as determine the threshold energy E0, from threshold to the upper limit that ensures the integral convergence due to attenuation. In case of atomic scattering, integrated cross-sections are usually determined through differential cross-sections; however, these are rather time-con- 
suming or impossible to measure at each of them in a wide range of dispersion angles [20-22].

In the assumption that distribution of colliding particles in regard to the relative motion energy $E$ is Maxwellian and independent of their internal energy (condition), the chemical reaction rate constant $k(T)$ under given temperature $T$ is related to its integrated cross section according to the formula

$$
k(T)=\frac{1}{k_{b} T}\left(\frac{8}{\pi \mu k_{b} T}\right)^{1 / 2} \int_{0}^{\infty} E^{1 / 2} \sigma(E) \exp \left(\frac{-E 0}{k_{b} T}\right) d E
$$

Then, if the assembly average solution of $\sigma(E)$ at the experimental range is known, it becomes possible to solve equation (10) and generalize this solution over the desired temperature range.

The advantage of this way is that an error in finding the integrated collision cross-section does not result in an increased relative error $\left|\frac{\delta(k(T))}{k(T)}\right|$ of the chemical reaction rate constant:

$$
\left|\lim _{M \rightarrow \infty} \frac{\int_{0}^{M} E^{1 / 2} \delta(\sigma(E)) \exp \left(\frac{-E 0}{k_{b} T}\right) d E}{\int_{0}^{M} E^{1 / 2} \sigma(E) \exp \left(\frac{-E 0}{k_{b} T}\right) d E}\right| \leq\left|\frac{\overline{\delta(\sigma(E))}}{\overline{\sigma(E)}}\right|
$$

where $\bar{X}$ - a designation of average size $X$.

Mathematically, the problem is formulated as the Fredholm integral equation of the first kind, with appropriate boundary and initial conditions. A challenge in solving the Fredholm integral equations of the first kind lies in the fact that basic data are not completely accurate (experimental data always have errors) and also there is a need to approximate a continuous problem with the discrete analogue. This results in having to solve ill-posed problems.

Different solution methods are used for ill-posed problems $[4,23,24]$. The most interesting ones from the practical point of view are methods based on approximation of an unstable exact problem with a parameter family of approximate stable problems and the choice of the parameter (regularization) depending on basic data perturbation level.

In solving equation (10), the process cross-section $\sigma(E)$ is found as a function of relative colliding particle motion energy $E$. For optimal solution of the Fredholm integral equation of the first kind, some a priori information on the collision cross-section is required $[23,24]$. Based on the review of the collision cross-section patterns , some direct experiments to measure the collision cross-section $\sigma(E)$ and the physical definition, we can assume the following a priori information on the collision cross-section $\sigma(E)$ : the function of the collision cross-section is positive, continuous, at least one time differentiable and bounded from above.

Based on a priori information on the collision crosssection, the Tikhonov's regularization was selected. The variational Tikhonov's regularization method is based on stabilization of deviation of the theoretical curve values $y 0(x)$ from the experimental curve values $y(x)=$ $y 0(x)+\delta(x)$ with the help of an auxiliary stabilizing functional $\Omega(x)$. This means that we solve a problem of $x$ minimizing of the variational Tikhonov's functional $F(x)$ :

$$
F(x)=\|A x-y\|^{2} \rho(x)+\alpha \Omega(x)
$$

with the stabilizing functional of the first-order Tikhonov's stabilizer:

$$
\Omega(x)=\left\|p(x) x^{2}+q(x) \frac{d x}{d y}\right\|
$$

where $\|\ldots\|$ - denotation of the norm ; $\alpha>0$ - regularization parameter; $p(x)>0, q(x)>0, \rho(x)>0$ - weight functions strengthening or weakening input to individual fields.

Applying the general Tikhonov's regularization theory for the Fredholm equation of the first kind (10), we shall write the variational Tikhonov's functional $F(x)$ as the following functional:

$$
\begin{aligned}
& F(T)= \\
& =\int_{T_{\min }}^{T_{\max }}\left(\frac{\frac{1}{k_{b} T}\left(\frac{8}{\pi \mu k_{b} T}\right)^{1 / 2} \int_{0}^{\infty} E^{1 / 2} \sigma(E) \exp \left(\frac{-E 0}{k_{b} T}\right) d E}{k_{\text {exp }}(T)}-1\right)^{2}+ \\
& +\alpha \Omega \\
& \text { where } \Omega=\int_{0}^{\infty}\left((\sigma(E))^{2}+\left(\frac{d \sigma(E)}{d E}\right)^{2}\right) d E ; \\
& \quad T_{\min }-\text { lower temperature limit; } \\
& \quad T_{\max } \text { - upper temperature limit; } \\
& k_{\text {exp }}(T) \text { - experimental dependence of the chem- } \\
& \text { ical reaction rate constant on a given temperat- } \\
& \text { ure range. }
\end{aligned}
$$

The following functions were selected as the weighting functions: $\rho(x)=\left(k_{\exp }(T)\right)^{-2}, q(x)=1, p(x)=1$. The weighting functions were selected by the principle of uniform approximation to the experimental value.

To select the regularization parameter $\alpha$, the discrepancy principle was used. For this purpose, equation $\delta^{2}=\left\|A x_{\delta}^{\alpha}-y\right\|^{2}$ ( was solved by the golden section method [7]. It is the equation: $\delta$ - experimental error; $x_{\delta}^{\alpha}$ - function that realizes the minimum functional with given regularization parameter $\alpha$.

Such an approach allowed avoiding undesirable extremes when choosing the regularization parameter $\alpha$ [24]. The rate constant values obtained in each of the original papers (Table 1) were treated as random values to which statistical data processing was applied.

Thus defined sets of chemical reaction rate constants (for all accounted papers of Table 1) were processed by the least-square method in the temperature range of $1,000 K$ to $5,000 K$. As a result, it was found that average chemical reaction rate constant values could be functionally represented as the following approximation $1.58 \cdot 10^{26} T^{-8.66} \exp \left(-\frac{60142}{T}\right), \mathrm{cm}^{3} / \mathrm{sec}$ over the temperature range of $1,000 K$ to $5,000 K$. The dispersion value was 0.5 .

The following values were found by selecting of model parameter values in the temperature range of $1,000 \mathrm{~K}$ to $5,000 K$ for the above models (I-III): 
The first model: gas-dynamic radius is $R^{2}=\left(R_{C_{H_{4}}}+\right.$ $\left.R_{N_{2}}\right)^{2} . \quad R_{C_{4}}$ and $R_{N_{2}}$ is gas-dynamic radius for methane and nitrogen. According to [25], gas-dynamic radius were taken 3.758 and $3.798 \stackrel{\circ}{\mathrm{A} m}$ as for methane and nitrogen, respectively. The threshold energy will be $E 0=41,477 \mathrm{~K}$. The collision cross-section has the dimension of $(\stackrel{\circ}{A} m)^{2}$.

The second model: the gas-dynamic radius is the same as in the first model. The threshold energy is $E 0=$ $37,812 \mathrm{~K}$. The collision cross-section has the dimension of $(\stackrel{\circ}{A} m)^{2}$.

The third model: $B=8.1 \cdot 10^{9}, l=3.53, r=62.57$. The threshold energy is $E 0=44,560 \mathrm{~K}$. The collision cross-section has the dimension of $(\stackrel{\circ}{A m})^{2}$.

The solution of the integral equation using the above method is shown in Fig. 2. The threshold energy is $E 0=$ $44,560 \mathrm{~K}$. The collision cross-section has the dimension of $(\stackrel{\circ}{A} m)^{2}$.

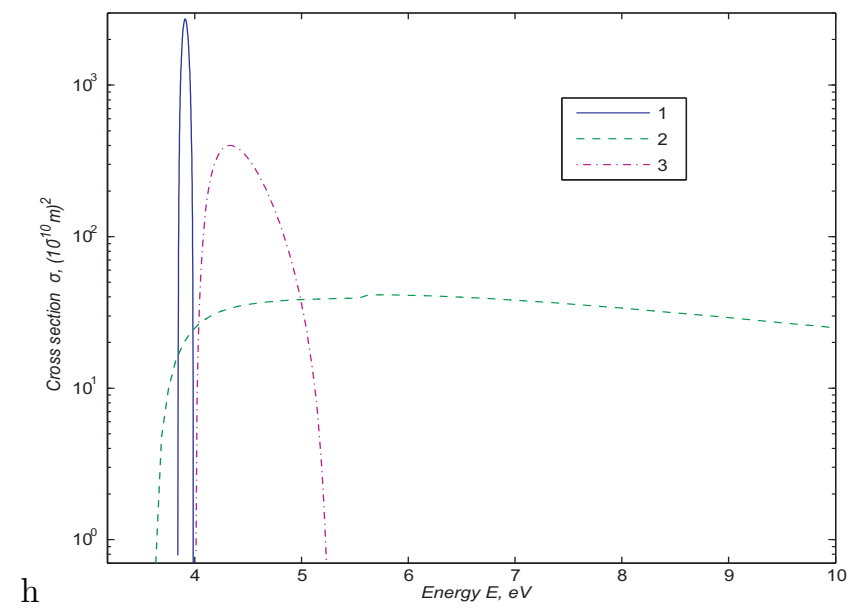

Figure 2. The find of integrated cross-section depending on forward energy by used solution of the integral equation (10): 1 $\mathrm{CH}_{4}+\mathrm{M} \rightarrow \mathrm{CH}_{3}+\mathrm{H}+\mathrm{M}, 2-\mathrm{CH}_{3}+\mathrm{M} \rightarrow \mathrm{CH}+\mathrm{H}_{2}+\mathrm{M}, 3-$ $\mathrm{CH}_{3}+\mathrm{M} \rightarrow \mathrm{CH}_{2}+\mathrm{H}+\mathrm{M}$.

The models can be conveniently analyzed on the basis of the error function, which can be defined as $f_{\text {error }}(T)=\left(\frac{k(T)}{k_{e x p}(T)}-1\right)^{2}$ (Fig. 3) and the mean error value Error $=\frac{1}{T_{\max }-T_{\min }} \int_{T_{\min }}^{T_{\max }} f_{\text {error }}(T) d T$. The average error was $72 \%, 89 \%, 35 \%$, and $34 \%$ for Models I to III and for the solution of integral equations, respectively.

The larger errors for Models I and II as compared with the error for Model III or the error in the solution of the integral equation results from the fact that the forms of the collision cross-sections obtained by the solution of the integral equation (Fig. 2) and by selection of parameters for the above-threshold maximum cross-section model accounting for the initial cross-section growth and its reduction to zero at high energies are fundamentally different from the cross-section used in Models I and II.

An indirect confirmation of the influence of the highenergy wing function of the methane molecule distribu-

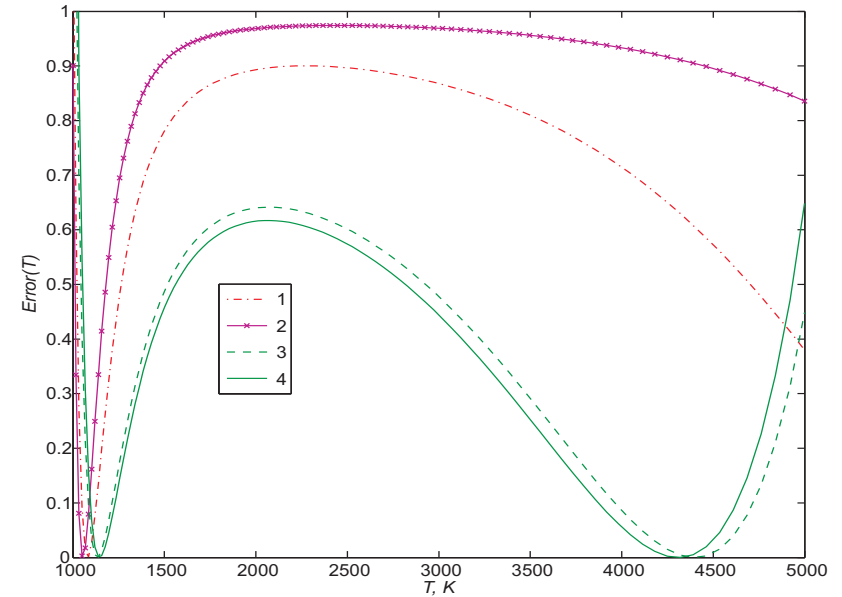

Figure 3. The function of Error for: 1 - Model I; 2 - Model II; 3 - Model III; 4 - The integrated section to be found by used of the integrated equation (10). Chemical reaction $\mathrm{CH}_{4}+\mathrm{M} \rightarrow$ $\mathrm{CH}_{3}+\mathrm{H}+\mathrm{M}$.

tion function on the chemical reaction rate can be found in paper [46]. In this work, Monte Carlo simulation of methane decomposition $\mathrm{CH}_{4}+\mathrm{Ar} \rightarrow \mathrm{CH}_{3}+\mathrm{H}+\mathrm{Ar}$ was carried out. It was shown that the radical decomposition rate $\mathrm{CH}_{4}$ was especially high in the initial period $t<3 \cdot 10^{-9}$, sec, then decreasing significantly. The authors [46, chap. 7] explain this by the fact that the initial period of the high wing of the methane molecule distribution function has a significant impact upon the reaction rate, and diabatic process of energy transfer from the argon atom to the methane molecule occurs.

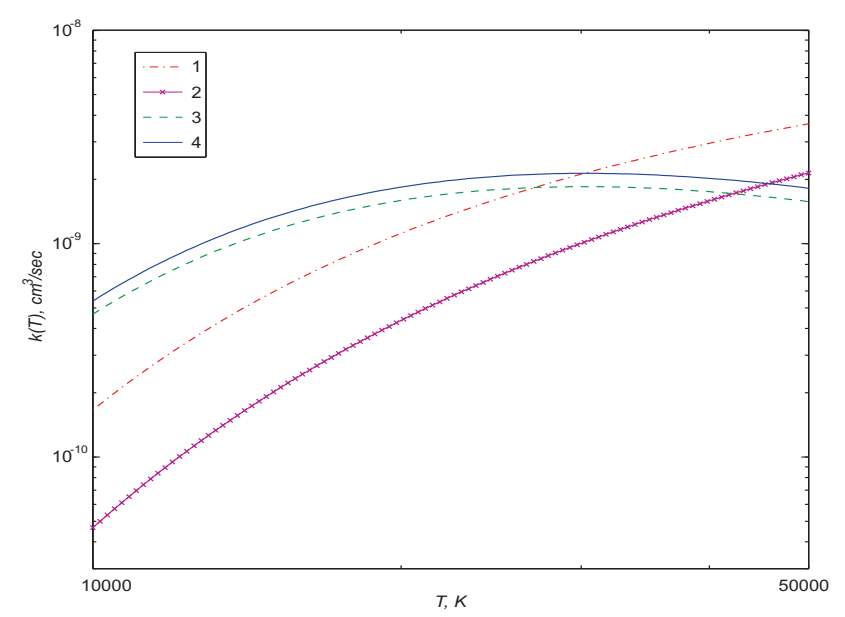

Figure 4. Approximation of the chemical reaction rate constant $\mathrm{CH}_{4}+\mathrm{M} \rightarrow \mathrm{CH}_{3}+\mathrm{H}+\mathrm{M}$ to area from 10,000 to $50,000 \mathrm{~K}: 1$ - Model I; 2 - Model II; 3 - Model III; 4 - The integrated section to bee found by used of the integrated equation (10).

The obtained values of integrated cross-sections allowed calculating the chemical reaction rate constants over a wide temperature range. The results of approximation of the Arrhenius dependence of the calculated chemical reaction rate constants for the temperature ranges of $1,000-5,000 K$ and $10,000 K-50,000 K$ are shown in Table 2.

In addition to the above methane dissociation reaction, the integral equation was used to find collision cross-sections for reaction $\mathrm{CH}_{3}+\mathrm{M} \rightarrow \mathrm{CH}_{2}+\mathrm{H}+\mathrm{M}$, $\mathrm{CH}_{3}+\mathrm{M} \rightarrow \mathrm{CH}+\mathrm{H}_{2}+\mathrm{M}$, where the third object is also assumed to be a nitrogen molecule. For these reac- 
tions, the threshold energy was $46,420 \mathrm{~K}$ and $42,070 \mathrm{~K}$, respectively. The integrated collision cross-sections profiles are presented in Fig. 2. Due to decreasing degrees of freedom of basic and final products and thus increasing rates of vibrational degree normalization for these reactions, the cross- section profile has a more traditional form of the attenuation of the tail section of the integrated collision cross-section.

Table 2. The results of approximation $A T^{n} \exp \left(-\frac{E a}{T}\right)$ for the chemical reaction rate constant of second-order $\mathrm{CH}_{4}+\mathrm{M} \rightarrow$ $\mathrm{CH}_{3}+\mathrm{H}+\mathrm{M}, \mathrm{CH}_{3}+\mathrm{M} \rightarrow \mathrm{CH}_{2}+\mathrm{H}+\mathrm{M}, \mathrm{CH}_{3}+\mathrm{M} \rightarrow$ $\mathrm{CH}+\mathrm{H}_{2}+\mathrm{M}$, depending on the temperature range. Temperature Range, $K: 1-1,000 \ldots 5,000 K, 2$ - 10,000...50,000 $K$

\begin{tabular}{|c|c|c|c|c|}
\hline Reaction & $A, \mathrm{~cm}^{3} / \mathrm{sec}$ & $n$ & $E a, K$ & note \\
\hline$C H_{4}+M \rightarrow$ & $4.42 \cdot 10^{-02}$ & -1.49 & 45,321 & 1 \\
\cline { 2 - 5 }$\rightarrow C H_{3}+H+M$ & $5.04 \cdot 10^{-02}$ & -1.50 & 45,377 & 2 \\
\hline$C H_{3}+M \rightarrow$ & $1.42 \cdot 10^{-04}$ & -0.85 & 48,040 & 1 \\
\cline { 2 - 5 }$\rightarrow C H_{2}+H+M$ & $5.27 \cdot 10^{-02}$ & -1.48 & 51,072 & 2 \\
\hline$C H_{3}+M \rightarrow$ & $3.86 \cdot 10^{-09}$ & 0.07 & 42,491 & 1 \\
\cline { 2 - 5 }$\rightarrow C H+H_{2}+M$ & $5.27 \cdot 10^{-02}$ & -1.48 & 51,072 & 2 \\
\hline
\end{tabular}

\section{Conclusions}

The paper discussed possible methods of approximation of the chemical reaction rate constant for the range of values that lie outside of the experimental temperature range: direct approximation of chemical reaction rate constants obtained by processing experimental values; approximation based on an analytical model of dependence of the integrated process cross-section on energy; and approximation based on the direct solution of the chemical reaction rate constant equation with arbitrary dependence of the integrated process crosssection on energy based on experimental data array for the chemical reaction rate constants of the second-order reactions $\mathrm{CH}_{4}+\mathrm{M} \rightarrow \mathrm{CH}_{3}+\mathrm{H}+\mathrm{M}, \mathrm{CH}_{3}+\mathrm{M} \rightarrow$ $\mathrm{CH}_{2}+\mathrm{H}+\mathrm{M}, \mathrm{CH}_{3}+\mathrm{M} \rightarrow \mathrm{CH}_{2}+\mathrm{H}_{2}+\mathrm{M}$.

It was shown that a direct approximation of experimental data for the outside-of- experimental range of values can result in significant scatter of the chemical reaction rate constants, and hence can be only used with a small deviation from the experimental temperature range.

The possibility of approximation of the chemical reaction rate constant to the outside-of-experimental temperature range was shown by solving the equation of the first kind by Tikhonov's regularization method. This method allows estimating energy dependences of integrated cross-sections of chemical reactions and the threshold energy value and re-establishing the form of the integrated collision cross-section.

By using the calculated cross-section we can obtain estimated chemical reaction rate constants over a wide temperature range. On this basis, estimates of the rate constants for chemical reactions $\mathrm{CH}_{4}+\mathrm{M} \rightarrow \mathrm{CH}_{3}+\mathrm{H}+$ $\mathrm{M}, \mathrm{CH}_{3}+\mathrm{M} \rightarrow \mathrm{CH}_{2}+\mathrm{H}+\mathrm{M}, \mathrm{CH}_{3}+\mathrm{M} \rightarrow \mathrm{CH}+\mathrm{H}_{2}+$ $M$ were obtained over a wide temperature range. The obtained threshold energy values for the above reactions were: $44,560 K, 46,420 K, 42,070 K$; approximation of chemical reaction rates for the temperature range $T=$
1,000 $-5,000 K$ and $T=10,000-50,000 K$ to see on the Table 2 .

Future work it is the aim modeling of high-speed shocked flows of at least reaching physically consistent rates for $\mathrm{T}=10,000-100,000 \mathrm{~K}$ temperature range [2]. While it cannot really be claimed that such improved dataset is validated in such an extended temperature range (due to the scarcely available experimental data for such high temperature ranges), it is capable of providing more accurate simulations of high-speed shocked flows for this mixture.

\section{REFERENCES}

[1] S. Surzhikov, Radiative-convective heat transfer of a spherically shaped space vehicle in carbon dioxideHigh temperature. 2011, Vol.49, No.1, 92-107

[2] H.F. Nelson, C. Park, E.E. Whiting, Titan atmospheric composition by hypervelocity shock-layer analysis, Journal of Thermophysics and Heat Transfer, 1991, Vol.5, No.2, 157-165

[3] A. Tarantola, Inverse Problem Theory Society for Industrial and Applied Mathematics, Philadelphia, Society for Industrial and Applied Mathematics, 2005

[4] V.K. Ivanov, V.V. Vasin, V.P. Tanana, Theory of linear ill-posed problems and its applications, Netherlands, VSP, 2002.

[5] MatLab@http://www.mathworks.com/

[6] MatLab Optimization Toolbox@ http://www.mathworks.com/products/optimization/

[7] R.A. Waltz, J.L. Morales, J. Nocedal, D. Orban, An interior algorithm for nonlinear optimization that combines line search and trust region steps, Mathematical Programming, 206, Vol.107, No.3, 391-408

[8] By Autar Kaw, Egwu Eric Kalu, Numerical Methods with Applications: Abridged, Perfect-bound Paperback, 2-st ed, 2011.

[9] NIST Chemical Kinetics Database Standard Reference Database 17, Version 7.0 (Web Version), Release 1.6.4 Data Version 2012.02 http://kinetics.nist.gov/

[10] Kintech Lab Chemical Workbench(C) http://kintechlab.com/products/chemical-workbench/

[11] Reaction Design@ Chttp://www.ReactionDesign.com

[12] Kintecus@http://www.kintecus.com/

[13] S. Arrhenius, Z. Phys. Chem. 4226 (1889);Translated into English in Selected Readings in Chemical Kinetics edited by M.H. Back and K.J. Laidler Oxford, 1967.

[14] J. Wolberg, Data Analysis Using the Method of Least Squares: Extracting the Most Information from Experiments, Springer, 2005.

[15] C.F. Hausen,Apparent Activation Energy of Chemical Reactions at High Temperature, Phys. Fluids, 1969, Vol.12, 1127-1129

[16] N.G. Utterbak, Charge Transfer Cross Sections for $\mathrm{N}_{2}^{+}$ Ions Incident on $\mathrm{H}_{2}$ and $\mathrm{D}_{2}$ between 20 and $1000 \mathrm{eV}$, Phys. Rev. Lett., 1968, Vol.20, 1021-1023 
[17] N.G. Utterbak, G.H. Miller, Fast Molecular Nitrogen Beam, Phys. Rev. Lett., 1961, Vol.124, 1477-1480

[18] L.I. Kieffer, G.H. Dume, Electron Impact Ionization Cross-Section Data for Atoms, Atomic Ions, and Diatomic Molecules: I. Experimental Data, Rev. Mod. Phys., 1966, Vol.38., 1-4

[19] G.G. Chernyi, S.A. Losev, S.O. Macheret, B.P. Potapkin, Physical and Chemical Processes in Gas Dynamics : Cross-Sections and Rate Constants, Vol.1, American Institute of Aeronautics \& Astronautics, 2002

[20] S. Trajmar, D.F. Register, A. Chutjian, Electron scattering by molecules II. Experimental methods and data, Phys. Rep., 1983, Vol.97, 219-223

[21] M.J. Brunger, S.J. Buckman, Apparent Activation Energy of Chemical Reactions at High Temperature electron-molecule scattering cross-sections.I. Experimental techniques and data for diatomic molecules, Phys. Rep., 2002, Vol.357, 215-218

[22] Cross-Section Data, By editor Mitio Inokuti. Academic Press, INC, California, 1994

[23] A.N. Tihonov, V.Ya. Arsenin, Methods for solving illposed problems, Moscow, Nauka, 1986. (in Russian)

[24] A.B. Vasileva, N.A. Tihonov, The integral equations, 2-st ed, Moscow, Fizmatlit, 2002. (in Russian)

[25] Estimation viscosities and thermal conductivities of gases at high temperatures technical, Report NASA TR R-132, By Roger A. Svehla, (1962).

[26] D.L. Baulch, C.J. Cobos, R.A. Cox, R.F. Hampson , J.A. Kerr, J. Troe, R.T. Watson, C.J. Cobos, Evaluated Kinetic Data for Combustion Modelling. Suppl. I, J. Phys. Chem. Ref. Data., 1994, No.23, 847-851

[27] D.L. Baulch, C.J. Cobos, R.A. Cox, R.F. Hampson , J.A. Kerr, J. Troe, R.T. Watson, C.J. Cobos, Evaluated Kinetic Data for Combustion Modeling, J. Phys. Chem. Ref. Data., 1992, No.21, 411-415

[28] J. Warnatz, Rate coefficients in the $\mathrm{C} / \mathrm{H} / \mathrm{O}$ system, Chap. 5 in Combustion Chemistry W.C. Gardiner Jr. pub. Springer-Verlag, 1984

[29] T. Koike, M. Kudo, H. Yamada, Rate Constants of $\mathrm{CH}_{4}+\mathrm{M}=\mathrm{CH}_{3}+\mathrm{H}+\mathrm{M}$ and $\mathrm{CH}_{3} \mathrm{OH}+\mathrm{M}=$ $\mathrm{CH}_{3}+\mathrm{OH}+\mathrm{M}$ over $1400-2500 \mathrm{~K}$, Int. J. Chem. Kinet., 2000, No.32, 1-6

[30] J.H. Kiefer, S.S. Kumaran, Rate of $\mathrm{CH}_{4}$ Dissociation over 2800-4300 K: the Low-Pressure-Limit Rate Constant, J. Phys. Chem., 1993 , No.97, 414-418

[31] R.B. Klemm, J.W. Sutherland, M.J. Rabinowitz, P.M. Patterson, J.M. Quartemont, W. Tao, Shock Tube Kinetic Study of Methane Dissociation: 1726-2134 K, J. Phys. Chem., 1992, No.96, 1786-1789
[32] D.F. Davidson, M.D. DiRosa, A.Y. Chang, R.K. Hanson, C.T. Bowman, A shock Tube Study of Methane Decomposition using Laser Absorption by $\mathrm{CH}_{3}$, Symp. Int. Combust. Proc., 1992, No.24, 589-593

[33] P.H. Stewart, G.P. Smith, D.M. Golden, The Pressure and Temperature Dependence of Methane Decomposition, Int. J. Chem. Kinet., 1989, No21, 923-926

[34] P. Roth, ARAS-Messungen an Einigen Hochtemperatur-Kohlenwasserstoff-Reaktionen, Forsch. Ingenieurwes., 1980, No.46, 93-97

[35] K. Tabayashi, S.H. Bauer, The Early Stages of Pyrolysis and Oxidation of Methane, Combust. Flame., 1979, No.34, 63-67

[36] P. Roth, T.H. Just, Measurements of Some Elementary Hydrocarbon Reactions at High Temperatures , NBS Spec. Publ. (U.S.)., 1979, No.10, 1339-1342

[37] S.S. Penner, K.G.P. Sulzmann, W.M. Heffington, G.E. Parks, Shock Tube Studies of Methane Pyrolysis and Oxidation Kinetics, Arch. Termodyn. Spalania., 1978, No9, 340-344

[38] W.M. Heffington, G.E. Parks, K.G.P. Sulzmann, S.S. Penner, Studies of Methane-Oxidation, Kinetics Symp. Int. Combust. Proc., 1977, Vol.16, 99

[39] P. Roth, Th. Just, Methan hinter Stosswellen Ber. Bunsenges, Phys. Chem., 1975, Vol.79, 682-684

[40] W.C. Gardiner, J.H. Owen, T.C. Clark, Rate and Mechanism of Methane Pyrolysis from 2000 to 2700 K, Symp. Int. Combust. Proc., 1975, Vol.15, 377-378.

[41] C.T. Bowman, Non-Equilibrium Radical Concentrations in Shock-Initiated Methane Oxidation, Symp. Int. Combust. Proc., 1975, Vol.15, 43

[42] G.A. Vompe, Thermal Decomposition of Methane at Low Pressures and High Temperatures, Russ. J. Phys. Chem., 1973, Vol.47, 1396-1399

[43] R. Hartig, J. Troe, H.G. Wagner, Thermal Decomposition of Methane Behind Reflected Shock Waves, Symp. Int. Combust. Proc., 1971, Vol.13, 147

[44] A.M. Dean, G.B. Kistiakowsky, Oxidation of Carbon Monoxide/Methane Mixtures in Shock Waves, J. Chem. Phys., 1971, Vol.54, 1718-1725

[45] D.W. Placzek, B.S. Rabinovitch, G.Z. Whitten, Some Comparisons of the Classical RRK and the RRKM Theoretical Rate Formulations, J. Chem. Phys., 1965, Vol.43, 4071-4075

[46] L.S. Polak, Nonequilibrium chemical kinetics and its application , Nauka, Moscow, 1979 (in Russian) 